\title{
B wie backstage oder ein Blick hinter die Kongresskulissen
}

\section{Dominik Heim}

PD Dr. med., Venenzentrum Thun, Mitglied FMH

"Welcome back my friends to the show that never ends.» Mit diesem Satz, dem Titel einer dreiteiligen LP (long play, 1974) von Emerson, Lake and Palmer, der Progressive-Rock-Band der 70er Jahre, wurde ein internationaler Venenkongress in Zürich beworben (Bild). Und die friends kamen - zu Hauf! Aus feuerpolizeilichen Kapazitätsgründen des Veranstaltungsortes musste deshalb die Registration schon zwei Wochen vor dem Kongress geschlossen werden. Das war äusserst bedauerlich und hat nicht ganz zu Unrecht zu viel Unverständnis geführt: Wie er denn nun zu den Credits komme, die er für 2019 noch brauche, schrieb jemand in seiner Verzweiflung, und ob es dafür nun Wiedergutmachungscredits gäbe? Das erinnert an andere Gelegenheiten, wo ein Kongressteilnehmer von einem Bekannten angefragt wird, ob er ihm auch grad seine Credits vom Kongress mitbringen könne. Es gibt eine Gesellschaft in der Schweiz, die gar eine Bearbeitungsgebühr zur Vergabe erhebt. Credits sind also ein kontroverses Thema.

Und sie sind auch (indirekt) mit der Industrie verknüpft, ob man sich dessen eigentlich bewusst ist? Denn Kongresse ohne die Teilnahme der Industrie sind heutzutage (fast) ein Ding der Unmöglichkeit. Was aber das Sponsoring anbelangt, da beruft sich die Industrie auf Vorschriften von MedTech Europe und Swiss Medtech, die besagen, dass Veranstaltungen, die

Chirurgische Gemeinschaftspraxis Hohmad, Thun

heim.dominik[at]bluewin.ch irgendwelche Form von Unterhaltung anbieten, nicht zu unterstützen sind. Das gipfelt in folgender Absage zur Anfrage für ein Sponsoring von fünfhundert Franken für eine themenbezogene kulturelle Einlage mitten im wissenschaftlichen Programm eines Kongresses: "Da der Auftritt aber sehr kurz ist (15 Min) und den generellen wissenschaftlichen Inhalt dieser 2-tägigen Veranstaltung nicht beschädigt, können wir an der Veranstaltung immer noch teilnehmen.» Mischt sich da die Industrie in die Programmgestaltung ein? Dazu schrieb ein entrüsteter Kollege: «Es ist tragisch, wie sich die Industrie zunehmend hinter ihren eigenen Regeln versteckt und uns im gleichen Atemzug im klinischen Alltag immer mehr einschränkt.»

Doch auch auf medizinischer Seite ziehen dunkle Wolken auf. Ein Ordinarius schrieb zu seiner Absage für ein Referat und zur Rückfrage für einen anderen Vorschlag: «Es ist tatsächlich immer schwieriger, Kaderleute für zusätzliche Arbeit zu gewinnen. Der Leistungsdruck killt diese Bereitschaft.» Da ist es dann Balsam, in der Zusage für ein Referat von einem anderen Ordinarius lesen zu können: «Ich mache das natürlich gerne - vor allem, um selbst von Ihren Beiträgen zu lernen.» Das ist dann einfach toll! Und stimmt im ganzen Kontext wieder optimistisch, verhehlt aber nicht ganz den Eindruck, dass Kongress-Organisieren zunehmend schwieriger wird.

Vieles tut sich also backstage, ohne dass es auf der Bühne so richtig wahrgenommen wird. Ob die Credits und ihre Vergabe, die (sich selbst?) auferlegte Zurückhaltung der Industrie und die zunehmend schwierigere Rekrutierung einer prominenten faculty inhaltsvollere Kongresse bewirken, bleibt abzuwarten. Ich fürchte mich vor einer wissenschaftlichen Einöde! Auf Procida, einer kleinen Insel im Golf von Neapel, lag in der einfachen Pensione «La perla rosa» am Meer auf dem Nachttischli das Buch «Post office» (1971) von Skandalautor Charles Bukowski (1920-1994). Er schreibt in seiner Einleitung: «This is presented as a work of fiction and dedicated to nobody.» Diese Kolumne hier ist «non-fiction and dedicated to whom it may (nein, does!) concern»!

Bildnachweis Dominik Heim 